\title{
PERSPECTIVA HISTÓRICA E CONCEITUAL DA PROMOÇÃO DA SAÚDE
}

Elioenai Dornelles Alves ${ }^{1}$, Alejandrina Arratia ${ }^{2}$, Denise M.Guerreiro V. da Silva ${ }^{3}$

RESUMO: trata-se de uma reflexão sobre a promoção da saúde na tentativa de construir um marco conceitual para orientar o desenvolvimento de um projeto de pesquisa sobre o que as instituições de saúde de Florianópolis-SC fazem para promover a saúde das pessoas. Destaca-se no texto a abrangência conceitual da promoção da saúde, contrastando com o conceito de prevenção de doença. São abordados alguns aspectos históricos, com destaque especial para a Conferência de Ottawa. São apresentadas algumas ações de promoção da saúde e destaca-se o papel do enfermeiro nas discussões sobre o tema.

DESCRITORES: Saúde; Promoção da saúde; Enfermagem.

\section{HISTORICALAND CONCEPTUAL PERSPECTIVE ON HEALTH PROMOTION}

ABSTRACT: A reflection is developed on helth promotion as an effort to build a conceptual framework capable of instructing the development of a research proposal that intends to anser the question "what Florianópolis health institutions do in order to promote people's health?". The text emphasizes the broad conceptual basis of helth promotion as opposed to the concept of disease prevention. Some historical aspects are covered with particular focus placed on the Ottawa Conference; a few health promoting actions are presented and the nurse's role is affirmed along with debates on the theme. DESCRIPTORES: Health; Health Promotion; Nursing.

\footnotetext{
${ }^{1}$ Professor da Universidade de Brasília. Doutorando da Pós-Graduação em Enfermagem da Universidade Federal de Santa Catarina-UFSC.

${ }^{2}$ Professora da Pontifícia Universidade Católica de Chile. Doutoranda da Pós-Graduação em Enfermagem da UFSC.

${ }^{3}$ Professora da Universidade Federal de Santa Catarina. Doutoranda da Pós-Graduação em Enfermagem da UFSC.
} 


\section{ASPECTOS HISTÓRICOS}

As duas manifestações políticas consideradas da maior importância para o cenário político internacional, como marcos referenciais no estudo da promoção da saúde no mundo, foram: a Carta de Ottawa, como resultante da $1^{\text {a }}$ Conferência Internacional sobre Promoção da Saúde, realizada no Canadá em 1986, e a Carta de Adelaide, fruto da II Conferência Internacional sobre Promoção da Saúde, realizada na Austrália em 1988.

A Carta de Ottawa (1986) apresenta aspectos imprescindíveis para as ações básicas de saúde pela implicações filosóficas que tem com a promoção da saúde, dentre as quais salientamos a proposição de uma política de saúde pública; os esforços para que uma consciência holísitico-ecológica enseje ambientes favoráveis; a participação popular em saúde de forma justa e ética; o pensar em potenciais de recursos humanos e a reorientação dos serviços de saúde.

A Carta de Adelaide (1988), além de ratificar asa posições das duas conferências anteriores: Alma-Ata e Ottawa, trouxe como ponto de recomendação a saúde como direito humano e justiça humana, aspecto desafiador/ inovador para os países que assinaram as recomendações. Essa conferência aprofundou as discussões sobre a dimensão da visão holística em saúde, tendo contribuindo com temas como a interdisciplinaridade, transdisciplinaridade e interinstitucionalidade (referindo-se aos diferentes setores integrantes do sistema de saúde).

A Constituição Brasileira de 1988 assegurou a saúde como um direito de todos e um dever do Estado, trazendo em seu conteúdo político-ideológico parcela das recomendações propostas na Conferência de Alma-Ata ( OMS/UNICEF,1978), nas Ações Integradas de Saúde - AIS - (Brasil/Ministério da Saúde, 1984), na $8^{a}$ Conferência Nacional de Saúde (1986), no Sistema Unificado e Descentralizado de Saúde - SUDS- (Brasil/Ministério da Saúde, 1987) e no resultado das manifestações do movimento pela reforma sanitária em nosso país.

Nessa linha de pensamento, a $8^{\mathrm{a}}$ Conferência Nacional de Saúde, a Constituição Brasileira de 1988 e a Lei Orgânica de 1990 contribuíram para dar uma visão da dimensão político-ideológica e sócio cultural das mudanças tão necessárias e esperadas no quadro sanitário brasileiro. Essa visão passou a ser enfatizada nas lutas coletivas/comunitárias, ao invés de individuais/ corporativas; dá-se ênfase à promoção da saúde em detrimento da prevenção da doença. Merece destaque que as discussões sobre o controle social passaram a efetivar a participação da sociedade civil organizada nas comissões interinstitucionais de saúde, que começaram a ser implantadas no país.

Mello \& Garrison (1993, p.2-3) analisando este momento e as implicações dele para o contexto político brasileiro afirmam que: esta abordagem traz para a prática novas estratégias de trabalho e ações políticas, através de processos que podem ser de confrontos e/ou negociações, que conduzem a transformações estruturais de condutas e de pensamento...

Toda essa trajetória na busca do entendimento da promoção da saúde no Brasil foi concretizada nas discussões e implantação de um novo Sistema Único de Saúde (SUS), retratando a preocupação com o aspecto econômico dos resultados/produtos esperados, com o rigor teórico/prático de essência e com nova abordagem/tendência em saúde no rumo de novo paradigma.

A ampliação de órgãos formadores de recursos humanos para a saúde não acompanhou inicialmente essa proposta de mudança de visão na saúde, continuando a formação de profissionais voltados para a cura e o cuidado da doença em detrimento da promoção da vida.

Ampliando as discussões e propostas sobre promoção da saúde, merece ainda destaque a reunião ocorrida na Espanha em 1988, onde os países da América Central analisaram a Carta de Ottawa e as implicações relativas à adequação da proposta nela contida às realidades dos países participantes. As principais conclusões/ recomendações oriundas desse encontro enfatizam que a melhoria da qualidade de vida se dará no momento em que a ampliação do conceito de promoção da saúde for algo real, implicando a melhoria da infra-estrutura básica global e intersetorial; a participação popular em saúde, efetivando o controle social; a criação das cidades saudáveis; e uma chamada à interdisciplinaridade.

Com relação às limitações, dificuldades ou problemas que poderiam surgir para a aplicação das recomendações na promoção da saúde, estes mesmos países destacaram a falta de políticas saudáveis para superação dos problemas detectados; a manutenção da unidirecionalidade da promoção da saúde, passando a entender os problemas somente a partir da comunidade; a inexistência de tecnologia própria e adequada para o intercâmbio interisntitucional; a resistência dos trabalhadores e gestores da saúde; o enfoque desenvolvimentista da promoção da saúde entendido como fora da realidade.

Dessa reunião em Granada - Espanha (Oficina Regional Europea, 1988) saíram três propostas para o alcance dos objetivos da reunião: -desenvolver pesquisa partcipativa para contestar o enfoque e o eixo de que o conhecimento não parte da realidade no campo da ação comunitária; - a avaliação social dos serviços de saúde assim como as metodologias de estudo-trabalho e educação atenuariam os problemas detectados no campo da ação de reorientação dos serviços sanitários; igualmente a educação familiar favoreceria a criação de cidades saudáveis, assim como de estilos de vida e o descobrimento da capacidade de auto-gestão baseada na metodologia problematizadora. 


\section{CONCEPÇÕES DE PROMOÇÃO DA SAÚDE}

Tradicionalmente as ciências da saúde se associam à doença e dor, às suas causas, consequências e tratamentos, constituindo estes aspectos os focos de atenção destas disciplinas.

Nos últimos anos a sociedade tem mostrado crescente interesse na saúde, o que pode ser observado pelo aumento das atividades dirigidas a melhorar os níveis de saúde da população. Foi deste modo que o conceito básico de promoção da saúde começou a ser cada vez mais reconhecido como uma nova meta de saúde (Stachtchenko e o Jenicek, 1990).

Antes de abordar a conceitualização da promoção da saúde como tal, é preciso destacar que tem sido difícil ter consenso a respeito do uso de uma definição operacional do termo, dado que também não há acordo sobre o que se entende por saúde; há na atualidade grande diversidade de entendimento sobre este conceito. A saúde, para Wilson (1975), é um conceito relacionado à cultura de um povo; isto significa que ela não poderá ser definida em termos absolutos, mas em relação às características de cada povo e à própria intenção de alcançar uma definição em determinadas situações.

De acordo com a Organização Mundial da Saúde, apud Stachtchenko e Jenicek (1990) "Saúde é um estado de completo bem-estar físico, mental e social e não meramente a ausência de doença ou enfermidade". Esta definição assinala uma condição abstrata que visualiza a saúde como uma meta ideal, difícil de alcançar. Definições mais recentes, por outro lado, enfatizam as habilidades individuais de cada pessoa para que desenvolvem seu potencial. A saúde é vista como produto da vida cotidiana, convertendo-se em recurso de vida mediante a qual indivíduos, grupos e comunidade se podem adaptar e responder positivamente às trocas como meio, realizando suas próprias ambições. O conceito adquire conotação positiva, na qual são valorizados de recursos sociais, individuais e as capacidades físicas de todas as pessoas (Stachtchenko e Jenicek, 1990).

$\mathrm{Na}$ atualidade, a noção de saúde se move para uma visão ecológica e de interação entre o indivíduo e seu meio físico e social. Este conceito sócio-ecológico foi central para a promoção da saúde nos anos 80 , constituindo estratégia mediadora entre as pessoas e seu meio, combinando assim, a preferência pessoal com a responsabilidade social.

Diversos autores têm proposto e desenvolvido valiosos aportes em relação à promoção da saúde; incorporam, ao longo do tempo, aspectos que têm favorecido a ampliação do campo de estudo e têm facilitado a discussão em relação ao tema. Entre os mais importantes pode ser mencionada a possibilidade que oferece o processo de promoção da saúde aos indivíduos, grupos e comunidade na condução de um comportamento positivo para as saúde, permitindo exercer maior controle sobre fatores que a determinam, favorecendo, um estilo de vida mais saudável. A condição para alcançar tudo isto está baseada na necessidade de que os atores tomem o compromisso real de assegurar um ótimo estado de saúde.

Outro referencial importante na discussão de conceito de promoção da saúde é a Carta de Ottawa (1986, p.1) que a coloca como:

processo que confere às populações os meios de assegurar o máximo controle sobre a saúde de cada indivíduo e melhorá-la, (...), vai mais além da prestação de cuidados, (...). Exige a ação concentrada de todos: governantes, o setor saúde e outros setores sociais e econômicos, organizações voluntárias não governamentais, autoridades locais, indústrias e serviços. (...). Sua função não recai somente no setor sanitário, mas vai mais além dos modos de vida saudável, visam ao bemestar

Esta proposta da Carta de Ottawa tem influenciado o planejamento e desenvolvimento de definições de vários outros autores (Saunders, 1988; Stachtchenko e Jenicek, 1990; Giilis, 1995), que a têm utilizado como principal fundamento e guia.

O processo de promoção da saúde adquire certa complexidade que determina uma dificuldade para ser abordado com métodos analíticos e de uma forma sintética. Isto tem significado que para alcançar o êxito de suas metas são necessárias diversas estratégias que estão baseadas no comportamento humano, e que procuram dar resposta à dimensão social, sempre presente em situações de saúde e enfermidade.

Outros aspectos importantes em relação à conceitualização de promoção da saúde são assinalados por Stachtchenko e Jenicek (1990, p.54):

a promoção da saúde corresponde a um conceito integrativo de amplo alcance, colocando ênfase no estilo de vida, modificação e focalização na redução de riscos de saúde, como por exemplo, parar de fumar, diminuição de álcool, drogas, etc.

O Ministério da Saúde do Chile (Chile, 1993, p. 49) apresenta outra definição de promoção de saúde:

o processo de permitir às pessoas aumentar a capacidade de auto-cuidado e responsabilidade sobre sua saúde (...) o que significa melhorar o nível de saúde e contribuir para elevar a qualidade de vida, atuando diante daqueles fatores que limitam ou afetam esta condição.

Assis (1990) afirma que a promoção da saúde requer trabalho interdisciplinar. Nesta perspectiva, portanto, os problemas de saúde requerem ser compreendidos do ponto de vista de diferentes áreas do conhecimento, nas quais devem ser consideradas variáveis de índole biológica, 
psicossocial, cultural e ambiental. Esta concepção abre novos horizontes de conhecimentos, o que requer a aplicação de novas técnicas e destrezas que permitam abordar os constantes desafios. Desse modo, a participação de todos os setores, tanto políticos como educacionais e econômicos, em seus diferentes níveis de gestão, são vitais para favorecer a efetividade da promoção à saúde.

Para resumir, pode-se dizer que as diversas abordagens são coincidentes em determinar que o contexto no qual se desenvolve a promoção da saúde representa nova estratégia, dentro da saúde e do campo social. Todas as concepções de promoção da saúde apresentadas aqui destacam a capacidade para desenvolver habilidades sociais, relacionadas com o próprio indivíduo, com a comunidade e com o meio ambiente, nos aspectos políticos, legislativos e recursos administrativos. Dessa forma, a estratégia de promoção à saúde pode ser integrada intersetorialmente, alcançando eficácia mediante ensino transdisciplinar.

Outro aspecto também, enfatizado nas definições de saúde mais recentes, é o que se relaciona ao estilo de vida das pessoas, influenciado de forma direta pelos aspectos culturais de cada povo. Isto determina a constante necessidade de potencializar e ajudar cada pessoa e a comunidade a alcançar o estado ótimo de saúde. A promoção da saúde se converte, desse modo, em recurso para a vida cotidiana, desde uma concepção da saúde que considera as variáveis biológicas, psíquicas, sociais, culturais e ambientais.

Utilizando aspectos da comparação feita por Stachtchenko e Janicek (1990) entre promoção da saúde e prevenção da doença, são destacados alguns pontos considerados relevantes.

A saúde dentro do enfoque de promoção corresponde a um conceito positivo e multidimensional. O modelo de saúde é participativo e dirigido a toda população, considerando seu meio ambiente. Dirigi-se a amplos aspectos dela, envolvendo-os com a utilização de diversas estratégias complementares, facilitando e estimulando a participação da população. Nos programas participam variados grupos da sociedade, como organizações não profissionais, grupos cívicos locais, municipais, regionais e nacionais.

A promoção da saúde se preocupa com a saúde de forma ampla; sua tendência é favorecer a constituição de uma população saudável, colocando ênfase especialmente no estilo de vida.

A prevenção da doença corresponde à ausência da doença. O modelo de saúde é meramente médico. Dirigi-se só a grupos de risco da população, portanto se preocupa com aspectos concretos de patologia, utilizando estratégias específicas, diretivas e persuasivas. Os programas estão focalizados em indivíduos ou grupos de risco, neles trabalham profissionais da saúde de disciplinas específicas. A redução de risco é a base de muitos programas preventivos. Tais programas precisam, muitas vezes, de testes diagnósticos e põem especial ênfase na modificação, focalização e redução de riscos de saúde. A intervenção médica aqui referida é restringida e dirigida somente a uma população alvo.

\section{AÇÕES DE PROMOÇÃO DA SAÚDE}

A literatura aponta algumas ações, atividades e/ou estratégias que estão envolvidas na promoção da saúde. Os autores: Saunders, 1988; Alves et al, 1992; Nutbeam, 1988; Mello e Garrison, 1993; Alves e Wright. 1994; Fernandez e Regules, 1991; King, 1994, procuram ressaltar a importância da contextualização das atividades propostas e destacam a participação de diferentes instituições nos programas, além das instituições de saúde. A participação comunitária é considerada fundamental em todos os estágios: no planejamento, na construção, na implementação e na avaliação, requerendo, necessariamente, o auto desenvolvimento da comunidade.

Promoção da saúde envolve atividades que incluem educação em saúde, manutenção e proteção, desenvolvimento da comunidade e do meio ambiente, pesquisas e políticas de saúde pública. Estas atividades para King (1994), levam a uma visão de que promoção da saúde é meio para um fim, uma entidade distinta no cuidado saúde de modo que assegure saúde para todos.

As ações de promoção da saúde para Nutbeam (1988) são tanto em nível individual quanto coletivo, sendo que a contribuição destes enfoque deve ser mantida em qualquer programa. Outro ponto relevante para o autor é que qualquer programa de promoção de saúde deve prever sua assimilação posterior pela comunidade; isto tem início com a participação da comunidade em todos os níveis do programa. Nutbeam (1988) ainda relaciona atividades bastante abrangentes, tais como: formação e desenvolvimento pessoal que inclui conhecimento e informação das causas e determinantes de saúde; serviços para a população, tais como vacinas, planejamento familiar, ajuda para deixar de fumar, perder peso, manterse em forma; informação e educação pelos meios de comunicação: marketing social e informação mediante notícias, ensino a distância pelo rádio e $\mathrm{TV}$; desenvolvimento organizativo com promoção da saúde entre seus próprios membros; medidas ambientais tais como: água potável, melhor planejamento de casas populares, rodovias mais seguras; atividades econômicas e reguladoras com medidas fiscais, legislação, controle do uso de agrotóxicos; e colaboração intersetorial: comércio, indústria, agricultura, transporte.

Dentre as ações de promoção da saúde contidas na Carta de Ottawa (1986) estão propostas atividades como: coordenação de encaminhamentos legais, medidas fiscais, taxação e mudanças organizacionais; proteção dos ambientes naturais; condições de vida e de trabalho seguras, estimulantes, prazerosas e agradáveis; participação efetiva 
e concreta da comunidade na fixação de prioridades, tomada de decisões e elaboração de estratégias de planificação para alcançar melhor nível de saúde; capacitar pessoas em todos os seus estágios de desenvolvimento para enfrentar doenças crônicas, agudas, acidentes e ferimentos; abrir canais entre o setor saúde e componentes sociais, políticos, econômicos e meio ambiente; atenção as pesquisas em saúde e mudanças na educação continuada.

Outros autores, que abordam ações de promoção da saúde como Edelman e Mandle (1994), referem que para que as pessoas se mantenham saudáveis são necessárias duas diferentes estratégias: - passivas, em que o cliente é recipiente das atividades, como, por exemplo, o acréscimo e vitamina D ao leite; e - ativas, em que o cliente deve estar pessoalmente envolvido, como, por exemplo, em exercícios diários, programas de controle de estresse, etc. É importante ressaltar, nessa colocação de Edelman e Mandle, que as atividades que eles propõe como passivas na realidade também requerem participação do cliente enquanto cidadão que reivindica seus direitos, que participa direta ou indiretamente das decisões políticas. Nesse sentido, não concordamos com a idéia de passividade na promoção da saúde, embora reconheçamos que ainda nos deparamos com tal concepção. Numa perspectiva de ações de promoção da saúde, acreditamos que todas as atividades devem contar com a participação comunitária representada pelas pessoas que a constituem.

No Brasil, a Reforma Sanitária, que projetou o Sistema Único de Saúde, colocou um deslocamento do poder político em direção às camadas populares, e passou a Ter participação institucionalizada. Nessa reforma, há o reconhecimento de que saúde é a expressão das modalidades de organização social e econômica, assumida como dever do Estado. No entanto, não são colocadas claramente as atividades de promoção da saúde, requerendo ainda muitas discussões entre os atores envolvidos no processo.

Na porposta dae Alves e Wright (1994) e Alves et al (1992), a participação efetiva do componente popular busca legitimar duas alternativas inovadoras para a academia e para a comunidade: uma ligada à pesquisa, introduzindo o paradigma promocionista da saúde (Carta de Ottawa, 1986) e crítico/holística (Wright, 1990), na busca da formação de profissionais de saúde que rspondam, de forma satisfatória, às necessidades da população . A outra, busca legitimar a participação popular na democratização e controle social das questões do ensino-serviço, saúde/doença e demanda social reprimida preconizadas na Constituição do Brasil de 1988.

A promoção da saúde tem provocado algumas discussões na enfermagem, que tem assumido iniciativas neste sentido. A enfermagem em nível mundial tem sido vista como profissão envolvida com a promoção da saúde; isto é evidenciado pelo interesse demonstrado por várias instituições em envolver enfermeiras nos programas de promoção, como por exemplo, a OMS que tem convidado as enfermeiras para serem líderes em movimentos de promoção da saúde, ao que elas tem respondido positivamente. A Associação de Enfermagem no Canadá tem reivindicado que a promoçaõ da saúde seja um território da enfermagem. No Reino Unido as organizações profissionais de enfermagem têm incluído a promoção da saúde nas recomendações de suas políticas. Também nos Estados Unidos têm havido movimentos para que seja colocada como prioridade nas pesquisas de enfermagem; em 1990, a American Nurses's Association (ANA) estabeleceu uma agenda nacional para a reformulação da saúde, com enfoque todo voltado para a promoção da saúde (Edelman e Mandle, 1994; Gillis, 1995).

O papel do enfermeiro na promoção da saúde no Brasil é questão que tem promovido poucas reflexões, não nos parecendo haver posição ainda muito clara da profissão a respeito dela, apesar de alguns enfermeiros não estarem só participando efetivamente dos programas de promoção da saúde, como também têm tomado a iniciativa, antecipando os requerimentos das mudanças na sociedade. Pareira (1992) numa reflexão sobre a responsabilidade individual ou social na promoção da saúde e prevenção de doenças, defende que:

cabe ao profissional refletir quanto às forças sociais que limitam as condutas de saúde da população. Através do entendimento crítico quanto ao contexto social, o enfermeiro manterá sua atuação não mais focalizada em uma perspectiva micro, mas favorecerá o envolvimento profissional em questionamentos mais abrangentes, e soluções alternativas poderão ser alcaçadas.

Trabalhos de pesquisa sobre promoção da saúde no Brasil ainda são escassos, porém trabalhos como o do grupo de Paranoá - cidade satélite do Distrito Federal (Alves e Wright, 1994), de um grupo de enfermeiros de Ribeirão Preto (Mendes, 1995), o projeto de pesquisa do qual o presente artigo faz parte (Trentini e Paim, 1993), têm trazido e trarão muito mais, sem dúvida, contribuições essenciais nas discussões do papel do enfermeiro na promoção da saúde.

Finalmente, gostaríamos de enfatizar que o papel do enfermeiro na promoção da saúde, em face principalmente das escassas discussões sobre o tema, ainda não é consenso entre os enfermeiros. Delaney (1994) defende que os limites da atuação da enfermagem na promoção da saúde ainda não estão claros, e que isto não é o mais importante. Acredita que a colaboração entre as disciplinas e a co-responsabilidade da comunidade são os princípios-chave da promoção da saúde. É justamente esse o ponto que consideramos fundamental nas discussões de promoção da saúde, o de que nenhuma profissão, isoladamente, poderá abranger/resolver as questões envolvidas no conceito, pois, tal como a entendemos, ela só existe numa perspectiva transdisciplinar. 


\section{REFERÊNCIAS}

1. ALVES, Elioenai D. et al. Os promotores de saúde-nutrição na comunidade do Paranoá DF. In: ENCONTRO INTERNACIONAL DE SAÚDE, 1, 1992, Santa Maria. Tema Livre... Santa Maria: UFSM, 1992.

2. ALVES, Elioenai D., WRIGHT, Maria da G. M. Estudo da promoção integral da saúde-nutrição da comunidade de Paranoá-DF. Brasília: CNPq, 1994. Projeto Integrado de Pesquisa, Relatório Técnico, 3 etapa, $n^{\circ}$ 500833/92.

3. ASSIS, Luiz F.S. Multidisciplinaridade, interdisciplinaridade, transdisciplinaridade?, Brasília: GT, PADCT/CIAMB 1990.

4. BRASIL, Ministério da Saúde. Ações Integradas de Saúde. Brasília: Centro de Documentação, 1984.

5. ___. Sistema Unificado e Descentralizado de Saúde. Brasília: Centro de Documentação, 1987.

6. CARTA de Ottawa. In: CONFERÊNCIA INTERNACIONAL SOBRE PROMOÇÃO DA SAÚDE, 1, 1986, Ottawa. Washington: OMS, 1986.

7. CARTA de Adelaide. In: CONFERÊNCIA INTERNACIONAL SOBRE PROMOÇÃO DA SAÚDE, 2, 1988, Adelaide. Washington: OMS, 1988.

8. CHILE. Ministério de la as Salud. De consultorio a centro de la salud. Santiago, 1993.

9. CONFERÊNCIA NACIONAL DE SAÚDE, 8, 1986, Brasília, Anais... Brasília: Ministério da Saúde, 1986.

10. DELANEY, Faith G. Nursing and health promotion: conceptual concerns. Journal of Advanced Nursing. Oxford, v.20, p.828-835, 1994.

11. EDELMAN, Carole L., MANDLE, Carol L. Health promotion - throughout the lifespan. St. Louis: Mosby, 1994.

12. FERNANDEZ, Luis Andres L., REGULES, Jose Manuel A., Promocion de la salud: un enfoque en la salud publica. Coleccion Sanidad Publica. N.2, p.23-46, 1991.

13. GILLIS, Angela. Exploring nursing outcomes for health promotion. Nursing Forum. V.30, n.2, p.5-12, Apr/Jun, 1995.

14. KING, Patricia M. Health promotion: the emerging frontier in nursing. Journal af Advanced Nursing, Oxford, v.20, p 209-218, 1994.

15. MELLO, Dalva A, GARRISON, Jane L. Reflexões sobre processos de ação e prática em promoção de saúde. Brasília: Universidade de Brasília, 1993.

16. MENDES, Iranilde J. Messias. Uma proposta holística de educação do adulto na perspectiva positiva de saúde. In: SEMINÁRIO NACIONAL DE SAÚDE DO ADULTO, 1 , 1995, Curitiba. Tema livre. Curitiba, 1995.

17. NUTBEAM, Don. La promocion de la salud en accionIdeas prácticas para llenar a cabo los programas. Salud, supl., feb., 1988

18. OFICINA REGIONAL EUROPEA. Servicio Andaluz de Salud, España. La promoción de la salud en accion: ideas prácticas para llevar a cabo los programas. Suplemento 32, Dirección General de Atención Primaria., 1988.

19. OMS/UNICEF. Declaração sobre cuidados primáriosde saúde. In: CONFERÊNCIA INTERNACIONAL SOBRE CUIDADOS PRIMÁRIOS DE SAÚDE, Alma-Ata, 1978.

20. PEREIRA, Rosane Carrion J. Promoção da saúde e prevenção de doenças: responsabilidade social. Revista da Escola de Enfermagem da USP, São Paulo, v. 26, n.3, p.345-350, dez., 1992.

21. SAUNDERS, Ruth P. What is health promotion? Health Education, Oct/Nov. 1988

22. STACHTCHENKO, S. Y. JENICEK, M. Conceptual differences between prevention and health promotion: research implications for community health programs. Canadian Journal of Public Halth, Ottawa, v.81, p.53-59, Jan/feb., 1990.

23. TRENTINI, Mercedes, PAIM, Lygia. Promoção: caminho apropriado à saúde. Florianópolis: Universidade Federal de Santa Catarina, 1933. (Projeto de pesquisa aprovado pelo $\mathrm{CNPq}$ ).

24. WILSON, M. Health is for people. London: DantonLougmann, 1975.

25. WRIGHT, Maria da Glória M. The need for a healthnutrition development paradigm. In: SEMINAR AT THE DANISH INSTITUTE FOR HEALTH AND NURSING RESEARCH, 1990, Copenhagen, 1990. 